\title{
Кинетика сорбции катиона лития свежеосажденным гидроксидом алюминия из природного рассола
}

\author{
(C) 2021 Рамазанов А.Ш. ${ }^{1,2}$, Свешникова Д.А. ${ }^{1}$, Атаев Д.Р. ${ }^{1}$ \\ ${ }^{1}$ Институт проблем геотермии и возобновляемой энергетики, филиал ОИВТ РАН, Махачкала \\ ${ }^{2}$ Дагестанский государственный университет, Махачкала
}

Поступила в редакцию 11.09.2020 г.

DOI: $10.17308 /$ sorpchrom.2021.21/3356

За двадцать лет XXI века потребление соединений лития в пересчете на карбонат лития, используемого в качестве стандартного рыночного товара, увеличилось в три раза, а цена с 2 до 8 долларов за килограмм. Более 35 \% соединений лития используется в производстве аккумуляторов и химических источников тока, около 32 \% в производстве керамики и стекла. С повышением спроса на литиевую продукцию принципиально изменилась сырьевая база, в настоящее время из гидроминерального сырья добывают около 70 \% соединений лития. Из крепких рассолов хлоридного натриевого типа американского континента карбонат лития получают по галургической технологии. В Китае из рассолов хлоридного магниевого и кальциевого типа с повышенным содержанием лития и общей минерализацией более 400 г/дм³ , литий извлекают использованием гранулированного сорбента разработанного ЗАО «Экостар-Наутех» (Новосибирск, Россия).

Работа посвящена исследованию кинетики сорбции $\mathrm{Li}^{+}$свежеосажденным гидроксидом алюминия из литийсодержащего природного рассола хлоридного натриевого кальциевого типа с минерализацией около 70 г/дм³.

Кинетические кривые адсорбции $\mathrm{Li}^{+}$на $\mathrm{Al}(\mathrm{OH})_{3}$ при различных температурах были обработаны уравнениями диффузионной и химической кинетики. Обработка экспериментальных данных с использованием расчетных кинетических диаграмм для смешанной диффузии выявила некоторое превалирование в смешанно-диффузионном механизме при 293 К внешней, а при 303 и 313 К внутренней диффузии.

Экспериментальные данные также были обработаны по уравнениям псевдо-первого и псевдовторого порядков, используемых для описания химической кинетики. Сравнение результатов показывает, что при 293 К значение расчетной величины сорбции дает лучшее совпадение с экспериментальной при использовании модели псевдо-первого порядка при практически одинаковых коэффициентах корреляции $\mathrm{R}^{2}$ для обеих моделей. При температурах 303 и 313 К значение величины сорбции лучше совпадает с экспериментальной при использовании модели псевдо-второго порядка. В пользу химической кинетики данного процесса в интервалах температур 293-303 и 303-313 К свидетельствуют энергии активации 94 и 46 кДж/моль соответственно.

Ключевые слова: природный рассол, литий, гидроксид алюминия, сорбция, кинетика.

\section{Введение}

В XXI веке наблюдаются изменения в структуре и увеличении объема потребления литиевых соединений во всех областях их применения. Основными областями применения их наряду с атомной и военной техникой являются производ- ство: литий-ионных батарей, специального стекла и керамики, катализаторов, консистентных смазок, сверхлегких сплавов, электролиз алюминия $[1,2]$.

В США, Чили, Боливии и Аргентине соли лития получают из рассолов хлоридного натриевого типа, в которых суммарное содержание $\mathrm{Mg}^{2+}$ и $\mathrm{Ca}^{2+}$, условно обозначаемой $\mathrm{C}_{\text {щз, }}$ к концентрации $\mathrm{Li}^{+}$: $R=\Sigma \mathrm{C}_{\text {щз }} / \mathrm{CLi}_{\mathrm{Li}}$ колеблется в пределах 3-24 
$[3,4]$. Технология основана на гелиоконцентрировании рассола в естественных бассейнах, что позволило выйти на высокие экономические показатели.

Рассолы с показателем $\mathrm{R}>24$ не могут быть переработаны по классической галургической технологии с многостадийной выпаркой, так как вначале необходимо очистить рассол от относительно большого количества ионов магния и щелочноземельных металлов $\left(\mathrm{Ca}^{2+}, \mathrm{Sr}^{2+}\right.$, $\mathrm{Ba}^{2+}$ ), карбонаты которых менее растворимы, чем карбонат лития. Извлечение $\mathrm{Li}^{+}$из рассолов данного типа может быть основано на различных селективных методах сорбции, ионного обмена и экстракции.

В монографии сибирских ученых Н.П. Коцупало и А.Д. Рябцева дан краткий обзор известных к 2008 году сорбционных методов извлечения лития из рассолов [3]. Отмечается, что наиболее селективными сорбционными свойствами по отношению к $\mathrm{Li}^{+}$из числа твердых сорбентов, кроме аморфного гидроксида алюминия, обладают простые и гидратированные оксиды марганца, титана, ниобия и других элементов, а также композиционные материалы на их основе.

Наибольший интерес у исследователей вызывают оксиды марганца и композиционные материалы на его основе [510]. Однако, эти сорбенты имеют дорогостоящую матрицу, и способ их синтеза достаточно трудоемок и энергоемок. Кроме того, композиционные материалы на основе оксидов марганца оказались пригодными в основном для извлечения $\mathrm{Li}^{+}$из морских и геотермальных вод, с минерализацией менее 40 г/дм ${ }^{3}$. В высокоминерализованных рассолах и при регенерации кислотой сорбенты на основе диоксида марганца не устойчивы.

В России литийсодержащие рассолы хлоридного натриевого типа, обогащенные $\mathrm{Mg}^{2+}$ и $\mathrm{Ca}^{2+}$ распространены на Северном Кавказе $(\mathrm{R}>60)$ и Восточной Сибири $(\mathrm{R}>200)$ [3]. Для извлечения $\mathrm{Li}^{+}$из рассолов данного типа не рентабельна галургическая технология, так как вначале необходимо очистить рассол от большого количества $\mathrm{Mg}^{2+}$ и $\mathrm{Ca}^{2+}$.

Известно [3,11-14], что селективными сорбентами по отношению к $\mathrm{Li}^{+}$лишенными недостатков характерных для сорбентов на основе диоксида марганца являются сорбенты на основе хлоридсодержащей разновидности двойного соединения алюминия и лития.

Для извлечения $\mathrm{Li}^{+}$из рассолов с высоким содержанием $\mathrm{Mg}^{2+}$ и $\mathrm{Ca}^{2+} 3 \mathrm{AO}$ «Экостар-Наутех» (Новосибирск, Россия) предложило гранулированный сорбент ДГАЛ-Cl на основе дефектной разновидности соединения $\mathrm{LiCl} 2 \mathrm{Al}(\mathrm{HO})_{3} \mathrm{nH}_{2} \mathrm{O}$ [11]. Промышленный синтез этого сорбента осуществлен китайской компанией «Foshan Electrical and Lighting Co., Ltd». В настоящее время этот сорбент компанией «Lan Ke Lithium Co., Ltd» (KHP) используется для производства литиевых продуктов из озерных рассолов [12].

Сорбент ДГАЛ-Сl имеет низкую рабочую емкость 2-3 мг/Г и предназначен для извлечения $\mathrm{Li}^{+}$из хлоридных магниевых и кальциевых рассолов с содержанием $\mathrm{LiCl}$ около 3 г/дм ${ }^{3}$ и общим солесодержанием более 400 г/дм³ ${ }^{3}$ Кроме того, для осуществления данной технологии требуется сложное и уникальное оборудование [12]. Указанные недостатки устранены, сорбционным извлечением $\mathrm{Li}^{+}$из слабых и крепких рассолов суспензией свежеосажденного гидроксида алюминия [15]. При этом образуется концентрат, основной фазой которого является хлоридсодержащая разновидность двойного гидроксида алюминия и лития $\mathrm{LiCl} \cdot 2 \mathrm{Al}(\mathrm{OH})_{3} \cdot 2 \mathrm{H}_{2} \mathrm{O}$. Следует отметить, что если механизм образования интеркаляционного соединения $\mathrm{Li}^{+}$с аморфным $\mathrm{Al}(\mathrm{OH})_{3}$ детально изучен и описан в монографии [13], то исследований, посвященных кинетике этого процесса ограничено $[15,16]$.

Целью данной работы является исследование кинетики сорбции $\mathrm{Li}^{+}$свежеосажденным гидроксидом алюминия из литийсодержащего природного рассола. 


\section{Экспериментальная часть}

Кинетику сорбции $\mathrm{Li}^{+}$свежеосажденным $\mathrm{Al}(\mathrm{OH})$ з изучали использованием самоизливающего рассола выработанного нефтяного месторождения (п. Берикей, Республика Дагестан) с содержанием, г/дм ${ }^{3}: \mathrm{Li}^{+}-0.042, \mathrm{Na}^{+}-24.0, \mathrm{~K}^{+}-0.59$, $\mathrm{Mg}^{2+}-0.27, \mathrm{Ca}^{2+}-1.4$; минерализацией 70 г/дм ${ }^{3}$ [17].

Так как в процессе сорбции $\mathrm{Li}^{+}$аморфным гидроксидом алюминия из макрокомпонентов конкурируют только $\mathrm{Mg}^{2+}$ из-за близости ионных радиусов [16], $\mathrm{Mg}^{2+}$ осаждали в виде гидроксида введением в рассол $10 \%$ избытка гидроксида кальция от стехиометрии:

$\mathrm{MgCl}_{2}+\mathrm{Ca}(\mathrm{OH})_{2} \rightarrow \mathrm{Mg}(\mathrm{OH})_{2} \downarrow+\mathrm{CaCl}_{2}$

Осаждение $\mathrm{Mg}^{2+}$ осуществляли при $\mathrm{pH}$ 10.5-11.0, температуре 293-333 К, продолжительности перемешивания рассола с известью в течение 60 минут. В результате такой обработки природный рассол очищается не только от $\mathrm{Mg}^{2+}$, но и от примесей $\mathrm{HCO}^{-}, \mathrm{Fe}^{2+}, \mathrm{Fe}^{3+}$, органических веществ [17].

Гидроксид алюминия для сорбции $\mathrm{Li}^{+}$ получали при температуре $20 \pm 1^{\circ} \mathrm{C}$ сливанием $1 \mathrm{M}$ раствора $\mathrm{AlCl}_{3}$ и $3 \mathrm{M}$ раствора $\mathrm{NaOH}$ :

$\mathrm{AlCl}_{3}+3 \mathrm{NaOH} \rightarrow \mathrm{Al}(\mathrm{OH})_{3} \downarrow+3 \mathrm{NaCl}$

Сорбцию $\mathrm{Li}^{+}$из очищенного от $\mathrm{Mg}^{2+}$ рассола объемом 2 дм³ осуществляли в реакторе с мешалкой введением свежеосажденного $\mathrm{Al}(\mathrm{OH})_{3} 2.9$ г из расчета обеспечения молярного отношения $\mathrm{Al}(\mathrm{OH})_{3} \mathrm{~K} \mathrm{Li}^{+}$в рассоле равное 1:3. Условия сорбции: $\mathrm{pH} 9.0 \pm 0.5$; температура 293-313 К; время до 3600 секунд.

$2 \mathrm{Al}(\mathrm{OH})_{3}+\mathrm{LiCl}+\mathrm{nH}_{2} \mathrm{O} \rightarrow \mathrm{LiCl} \cdot 2 \mathrm{Al}(\mathrm{OH})_{3} \cdot \mathrm{nH}_{2} \mathrm{O} \downarrow$

Сорбционную емкость $\mathrm{Al}(\mathrm{OH})_{3}(a, \mathrm{Mr} / \Gamma)$ оценивали определением содержания $\mathrm{Li}^{+}$ в объеме рассола до и после сорбции методом атомно-абсорбционной спектрометрии на приборе марки contraAA 700 (Analytik Jena, Германия).

\section{Обсуждение результатов}

Из рис. 1, на котором представлена зависимость сорбции $\mathrm{Li}^{+}$от времени, видно, что если скорость сорбции с повышением температуры в интервале 293313 К увеличивается, то со временем величина сорбции с повышением температуры уменьшается. Наблюдается частичная десорбция $\mathrm{Li}^{+}$с сорбента.

Для описания гетерогенных процессов используют уравнения диффузионной и химической кинетики. Когда обе кинетические модели с высокой точностью описывают процесс адсорбции, предполагается, что скорость адсорбции контролируется обоими механизмами.

Обычно используемый стандартный подход для описания кинетики адсорб-

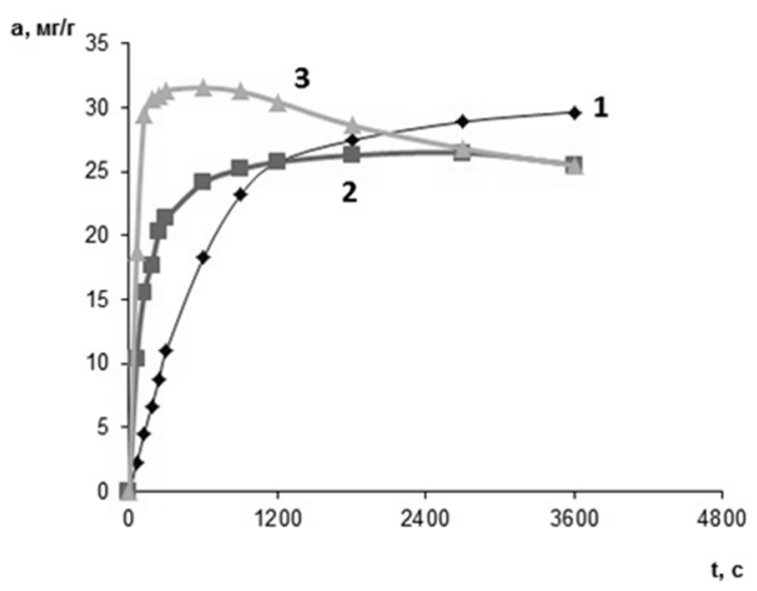

Рис. 1. Кинетические кривые адсорбции $\mathrm{Li}^{+}$ на свежеосажденном гидроксиде алюминия: 1 - 293 К; 2 - 303 К; 3 - 313 К

Fig. 1. Kinetic curves of $\mathrm{Li}^{+}$adsorption on freshly precipitated aluminium hydroxide: $1-293 \mathrm{~K} ; 2-303 \mathrm{~K} ; 3-313 \mathrm{~K}$ 
ции в диффузионной области заключается в использовании для описания внешней диффузии уравнения [18]:

$$
\ln (1-F)=-\frac{3 D_{i} t}{r \delta \Gamma},
$$

где $F=a_{t} / a_{e} ; D_{i}-$ коэффициент внешней диффузии, $\mathrm{cm}^{2} / \mathrm{c} ; t$ - время, с; $r$ - радиус частиц сорбента, см; $\delta$ - неподвижная пленка вокруг зерен сорбента, толщину которой принято считать $10^{-2}-10^{-3} \mathrm{~cm}$, $\Gamma=\mathrm{a}_{\mathrm{e}} / \mathrm{c}_{\mathrm{e}}-$ коэффициент распределения (ае - концентрация вещества в фазе сорбента, равновесная с концентрацией се). Для описания внутренней диффузии используется уравнение Бойда-Адамсона [19]:

$$
F=\frac{a_{t}}{a_{e}}=1-\frac{6}{\pi^{2}} \sum_{n=1}^{\infty} \frac{1}{n^{2}} \exp \left(-\frac{\bar{D} \pi^{2} n^{2} t}{r^{2}}\right),
$$

где $\bar{D}$ - коэффициент внутренней диффузии, $\mathrm{cm}^{2} / \mathrm{c} ; \mathrm{n}$ - ряд целых чисел $1,2,3,4,5 \ldots$

Для больших значений времени адсорбции из-за быстрого сходимости ряда можно обойтись только первым членом $(\mathrm{n}=1)$ и уравнение (2) принимает вид:

$$
F=1-\frac{6}{\pi^{2}} \exp \left(-\frac{\bar{D} \pi^{2} t}{r^{2}}\right)
$$

Использование в уравнении (1) параметра $\delta$ резонно подвергается критике [20], поскольку само существование такой пленки недостаточно обосновано, а ее толщина, которую непосредственно измерить нельзя, является условной математической величиной.

Вместе с тем, из экспериментальных данных по уравнениям (1) и (3) рассчитаны кинетические параметры адсорбции $\mathrm{Li}^{+}$на $\mathrm{Al}(\mathrm{OH})_{3}$. В расчетах использовалось значение радиуса $\mathrm{r}=3.5$ мкм, которое приводится в работе [21] для частиц гидроксида алюминия, полученного химическим способом из концентрированных растворов хлорида алюминия.

Одним из способов оценки долей внешней и внутренней диффузии в общем диффузионном процессе является вычисление так называемого коэффициента Био [22]:

$$
B i=\frac{D_{i} r}{\bar{D} \delta \Gamma}
$$

при этом в [22] отмечается, что процесс сорбции лимитируется внутренней диффузией при $\mathrm{Bi} \geq 50$, а при $\mathrm{Bi}<10$ - внешней.

Результаты расчетов кинетических параметров внешней и внутренней диффузии приведены в таблице 1, из которой видно, что при всех исследуемых температурах процесс сорбции $\mathrm{Li}^{+}$гидроксидом алюминия контролируется диффузионной кинетикой с преобладанием внешней диффузии.

Вместе с тем, обычно для характеристики диффузионной кинетики используют два параметра: вместо коэффициента внутренней диффузии используют коэффициент массопереноса вещества в жидкой фазе к поверхности сорбента $\beta$ и коэффициент внутренней диффузии $\bar{D}$. Следует отметить, что для получения достоверных экспериментальных данных определение коэффициента внутренней диффузии $\bar{D}$ проводится методом тонкого слоя при пропускании исходного раствора через ячейку с максимально высокой линейной скоростью. Коэффициент массопереноса $\beta$ может быть вычислен из «мгновенного проскока», наблюдаемого в начальный момент времени на кинетической кривой.

Таблица. 1. Диффузионные кинетические параметры, рассчитанные по уравнениям (1), (3), (4)

Table. 1. Diffusion kinetic parameters calculated using equations (1), (3), (4)

\begin{tabular}{|l|l|l|l|}
\hline $\mathrm{T}, \mathrm{K}$ & $\mathrm{D}_{\mathrm{i}, \mathrm{cm}^{2} / \mathrm{c}}$ & $\bar{D}, \mathrm{~cm}^{2} / \mathrm{c}$ & $B i$ \\
\hline 293 & $2.62 \cdot 10^{-7}$ & $2.11 \cdot 10^{-11}$ & 3.1 \\
303 & $2.21 \cdot 10^{-6}$ & $3.11 \cdot 10^{-11}$ & 5.1 \\
313 & $6.61 \cdot 10^{-5}$ & $2.00 \cdot 10^{-10}$ & 3.3 \\
\hline
\end{tabular}


Как отмечается в [20], внутридиффузионная кинетика имеет место в случаях, когда сопротивление в жидкой фазе мало $(\beta \rightarrow \infty)$. В этом случае скорость процесса является функцией безразмерного внутридиффузионного времени $T_{i}=\bar{D}_{i} t / r^{2}$. Внешнедиффузионная кинетика проявляется в тех случаях, когда $\bar{D}_{i} \rightarrow \infty$, а скорость сорбции зависит от безразмерного внешнедиффузионного времени $T_{e}=\beta t / \Gamma$ ( $Г$-коэффициент распределения). Критерием относительного вклада в кинетику сорбции обоих диффузионных механизмов является безразмерный параметр [20]

$$
H=T_{e} / T_{i}=\frac{\beta r^{2}}{\bar{D} \Gamma}
$$

При $\mathrm{H} \gg>1$ кинетика процесса лимитируется внутренней диффузией, а при $\mathrm{H} \rightarrow 0$ кинетика процесса контролируется внешней диффузией. Значения $\mathrm{H}$, лежащие между указанными значениями свидетельствуют о смешанной кинетике [20].

Учитывая, что чаще всего исследователи имеют дело со смешанной диффузией, использование модели для смешанной диффузии является общим подходом при теоретическом описании кинетических экспериментов.

Авторы в [20] приводят уравнение для смешанной кинетики, отмечая, что «концентрация в шаре радиусом $r$ при обмене со средой по закону

$$
\beta_{n}\left(c_{0}-c_{\text {nов }}\right)=D_{i}\left(\frac{\partial a^{(s)}}{\partial l}\right)_{l=r}
$$

( $\mathrm{c}_{0}=\mathrm{const} ; \mathrm{c}(1.0)=0 ; 1-$ расстояние от центра шара), характеризующему внешнедиффузионную часть кинетического сопротивления при линейной изотерме $a^{(s)}=\Gamma c_{\text {пов }}$, имеет вид»:

$$
\begin{array}{r}
\frac{a^{(s)}\left(\frac{l}{r^{\prime}} T\right)}{C_{o} \Gamma}=1-\frac{2 H r}{l} \sum_{i=1}^{\infty} \exp \left(-\alpha_{i}^{2} T\right) . \\
\frac{\alpha_{0}^{2}+(H-1)^{2}}{\alpha_{1}^{2}+H(H-1)} \sin \alpha_{i} \sin \alpha_{i} \frac{l}{r}(6)
\end{array}
$$

где $H=\beta r^{2} / \overline{D_{i}} \Gamma ; \alpha_{i}-i$-й корень трансцендентного уравнения $\alpha \operatorname{ctg} \alpha+H-1=0$.
Семейство теоретических кривых $F=f(T, H)$ для различных Н, отражающее уравнение (6), построенное в билогарифмической системе координат $(\lg F-\lg T)$, приведено в работе [20].

Предположим, что полученные нами экспериментальные зависимости $F=f(t)$ могут быть описаны теоретическими уравнениями для смешанной диффузии, приведенными в [20]. Сложность этой модели позволяет работать с аналитическими выражениями только для линейных частей изотерм.

Использование расчетных кинетических диаграмм, приведенных в [19], заключалось в наложении на эти теоретические кривые экспериментальных точек в координатах $\lg F-\lg t$. Установив, при каком значении Н теоретические и экспериментальные кривые совпадают, по разности между осями абсцисс $(\lg T-\lg t)$ теоретических и экспериментальных кривых, учитывая, что $\quad T=\frac{\beta \cdot t}{\Gamma} \quad$ определяли $\lg \frac{\beta \cdot t}{\Gamma}-\lg t=\lg \frac{\beta}{\Gamma}$. По величине $\lg \frac{\beta}{\Gamma}$ находили $\beta$, значение коэффициента внутренней диффузии $\bar{D}$ вычисляли по уравнению (5), используя значение Н, при котором теоретические и экспериментальные кривые совпадают

На рис. 2-4 и таблице 2 представлены результаты обработки экспериментальных данных с использованием расчетных кинетических диаграмм для смешанной диффузии и линейной изотермы.

Из таблицы 2 видно, что значения параметра Н меняются с ростом температуры от 8 до 100, что свидетельствует о некотором превалировании в смешаннодиффузионном механизме при 293 К внешней, а при 303 и 313 К внутренней диффузии, на это же указывают и значения параметров $\beta$ и $\bar{D}$.

Экспериментальные данные были обработаны также по уравнениям псевдопервого (7) и псевдо-второго (8) порядков, используемых для описания химической кинетики [23,24]. 


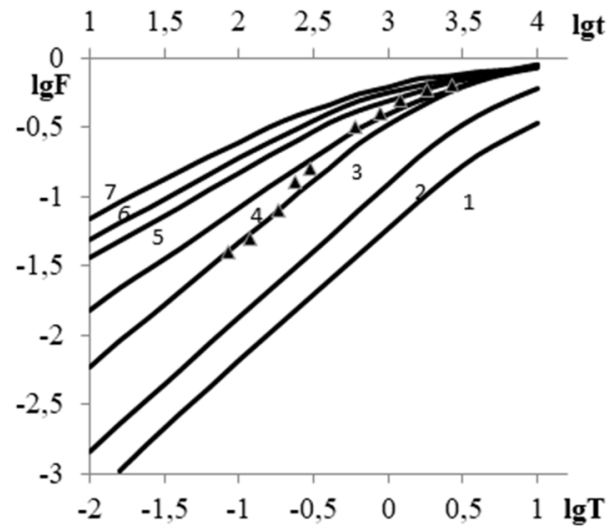

Рис. 2. Теоретические зависимости $\mathrm{F}(\mathrm{H}, \mathrm{T})$ при смешанно-диффузионной кинетике при значениях Н: $1-1 ; 2-2 ; 3-8$; $4-20 ; 5-63 ; 6-100 ; 7-\infty$ [12] и экспериментальные данные: адсорбция $\mathrm{Li}^{+}$на

$\mathrm{Al}(\mathrm{OH})_{3}$ при температуре $293 \mathrm{~K}$

Fig. 2. Theoretical dependences $F(H, T)$ for mixed-diffusion kinetics for different values of $\mathrm{H}: 1-1 ; 2-2 ; 3-8 ; 4-20 ; 5-63$ $6-100 ; 7-\infty$ [12] and experimental data: adsorption of $\mathrm{Li}^{+}$on $\mathrm{Al}(\mathrm{OH})_{3}$ at a temperature of $293 \mathrm{~K}$

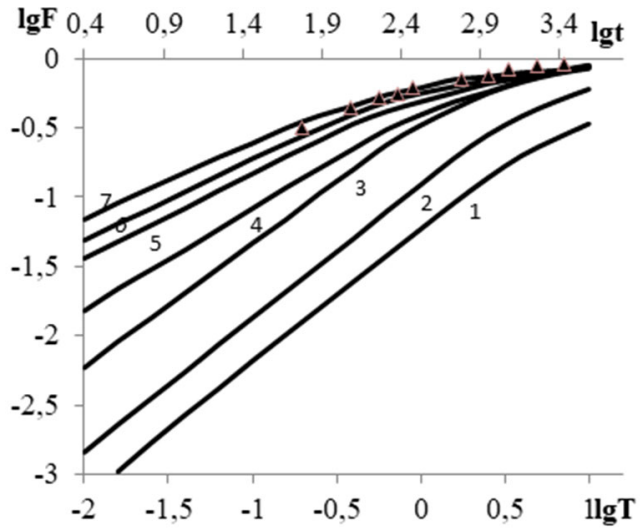

Рис. 3. Теоретические зависимости $\mathrm{F}(\mathrm{H}, \mathrm{T})$ при смешанно-диффузионной кинетике при значениях $\mathrm{H}: 1-1 ; 2-2 ; 3-8$; $4-20 ; 5-63 ; 6-100 ; 7-\infty$ [12] и экспериментальные данные: адсорбция $\mathrm{Li}^{+}$ на $\mathrm{Al}(\mathrm{OH})_{3}$ при температуре $303 \mathrm{~K}$.

Fig. 3. Theoretical dependences $F(H, T)$ for mixed-diffusion kinetics for different values of $\mathrm{H}: 1-1 ; 2-2 ; 3-8 ; 4-20 ; 5-63$; $6-100 ; 7-\infty$ [12] and experimental data: adsorption of $\mathrm{Li}^{+}$on $\mathrm{Al}(\mathrm{OH})_{3}$ at a temperature of $303 \mathrm{~K}$

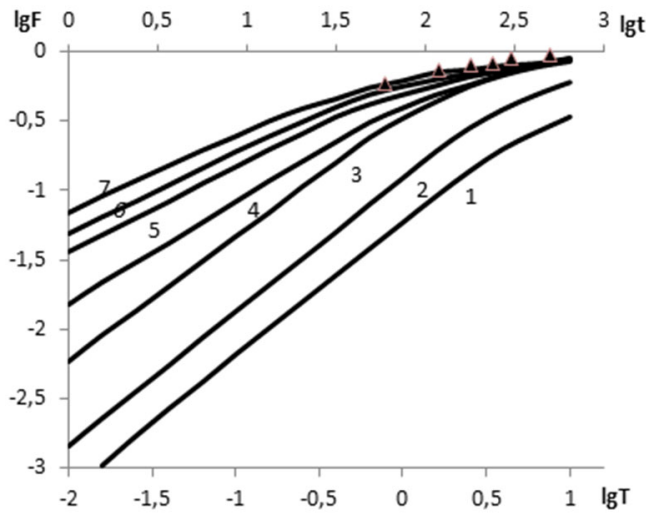

Рис. 4. Теоретические зависимости $\mathrm{F}(\mathrm{H}, \mathrm{T})$ при смешанно-диффузионной кинетике при значениях Н: $1-1 ; 2-2 ; 3-8 ; 4-20 ; 5-63 ; 6-100 ; 7-\infty$ [12] и экспериментальные данные: адсорбция $\mathrm{Li}^{+}$на $\mathrm{Al}(\mathrm{OH})_{3}$ при температуре $313 \mathrm{~K}$

Fig. 4. Theoretical dependences $\mathrm{F}(\mathrm{H}, \mathrm{T})$ for mixed-diffusion kinetics for different values of $\mathrm{H}$ : $1-1 ; 2-2 ; 3-8 ; 4-20 ; 5-63 ; 6-100 ; 7-\infty$ [12] and experimental data: adsorption of $\mathrm{Li}^{+}$on $\mathrm{Al}(\mathrm{OH})_{3}$ at a temperature of $313 \mathrm{~K}$

$$
\begin{aligned}
\ln \left(a_{e}-a_{t}\right) & =\ln a_{e}-k_{1} t \\
\frac{t}{a_{t}} & =\frac{1}{k_{2} a_{e}^{2}}+\frac{1}{a_{e}} t,
\end{aligned}
$$

где ае и ат - величина сорбции равновесной и в момент времени $t$ соответственно $(\mathrm{Mг} / \Gamma), \mathrm{k}_{1}\left(\right.$ мин $\left.^{-1}\right)$ и $\mathrm{k}_{2}\left(г \cdot \mathrm{M}^{-1} \cdot \mathrm{Mин}^{-1}\right)-к о н-$ станты скорости псевдо-первого и псевдо-второго порядков. Результаты приведены в таблице 3 .
Сравнение результатов (табл. 3) показывает, что при температуре 293 К значения арасч дают лучшее совпадение с экспериментальными аеэксп, при использовании модели псевдо-первого порядка при практически одинаковых коэффициентах корреляции $\mathrm{R}^{2}$ для обеих моделей. При температурах 303 и $313 \mathrm{~K}$ значение арасч лучше совпадает с аэксп при использовании модели псевдо-второго порядка. 
Таблица 2. Диффузионные параметры адсорбции $\mathrm{Li}^{+}$на $\mathrm{Al}(\mathrm{OH})_{3}$ при различных температурах

Table 2. Diffusion parameters of $\mathrm{Li}^{+}$adsorption on $\mathrm{Al}(\mathrm{OH})_{3}$ at different temperatures

\begin{tabular}{|c|c|c|c|}
\hline $\mathrm{T}, \mathrm{K}$ & $\beta, \mathrm{c}^{-1}$ & $\bar{D}_{i} \mathrm{~cm}^{2} / \mathrm{c}$ & $\mathrm{H}$ \\
\hline 293 & $9.75 \cdot 10^{-1}$ & $5.30 \cdot 10^{-11}$ & 8 \\
303 & 7.03 & $8.87 \cdot 10^{-12}$ & 100 \\
313 & 158.40 & $2.74 \cdot 10^{-11}$ & 100 \\
\hline
\end{tabular}

Таблица 3. Кинетические параметры реакции псевдо-первого и псевдо-второго порядков для $\mathrm{Li}^{+}$на $\mathrm{Al}(\mathrm{OH})_{3}$ при различных температурах

Table 3. Kinetic parameters of the reaction of pseudo-first and pseudo-second orders for $\mathrm{Li}^{+}$ on $\mathrm{Al}(\mathrm{OH})_{3}$ at different temperatures

\begin{tabular}{|c|c|c|c|c|c|c|c|}
\hline \multirow[t]{2}{*}{$\mathrm{T}, \mathrm{K}$} & \multicolumn{3}{|c|}{ Псевдо-первый порядок } & \multicolumn{3}{|c|}{ Псевдо-второй порядок } & \multirow{2}{*}{$\begin{array}{c}\mathrm{a}_{\text {эксп }}, \boldsymbol{\Gamma}\end{array}$} \\
\hline & $\mathrm{k}_{1}$, мин $^{-1}$ & $\begin{array}{l}\mathrm{a}_{\text {pacu, }}, \\
\mathrm{M \Gamma} / \Gamma\end{array}$ & $\mathrm{R}^{2}$ & $\begin{array}{c}\mathrm{k}_{2}, \\
\text { Г/МГ·Мин }\end{array}$ & $\begin{array}{l}a_{\text {pacy, }} \\
\mathrm{M \Gamma} / \Gamma\end{array}$ & $\mathrm{R}^{2}$ & \\
\hline 293 & $8.36 \cdot 10^{-2}$ & 27.4 & 0.988 & $2.21 \cdot 10^{-3}$ & 37.0 & 0.986 & 29.7 \\
\hline 303 & $1,59 \cdot 10^{-1}$ & 17.9 & 0.945 & $1.69 \cdot 10^{-2}$ & 29.0 & 0.999 & 28.5 \\
\hline 313 & $9,62 \cdot 10^{-1}$ & 22.4 & 0.950 & $1.52 \cdot 10^{-1}$ & 29.2 & 0.997 & 31.6 \\
\hline
\end{tabular}

Следует отметить, что часто зависимость $\ln \left(\mathrm{a}_{\mathrm{e}}-\mathrm{at}_{\mathrm{t}}\right)-\mathrm{t}$ носит мультилинейный характер, в результате чего невозможно описать всю кинетическую кривую уравнением псевдо-первого порядка. Кроме того, как указывается в $[25,26]$ уравнение модели псевдо-первого порядка может описывать кинетику диффузионных процессов.

Авторами работы [24] была предпринята попытка установления различия между диффузионной и химической моделями кинетики сорбции в системах, где уравнение Лагергрена удовлетворительно описывает экспериментальные данные.

Следуя рассуждениям, приведенным в [24], перепишем уравнение (3) в виде:

$$
\ln \left(a_{e}-a_{t}\right)=\ln a_{e}+\ln \frac{6}{\pi^{2}}-\frac{D \pi^{2}}{r^{2}} t
$$

и сравним его с уравнением (7) в аналогичной форме. Из этого сравнения видно, что существует разница между теоретической интерпретацией отрезка, отсекаемого от оси ординат в уравнении (9) $\left(\beta_{\mathrm{D}}\right)$ :

$$
\beta_{D}=\ln a_{e}+\ln \frac{6}{\pi^{2}}
$$

и в уравнении $(7)\left(\beta_{\mathrm{L}}\right)$ :

$$
\beta_{L}=\ln a_{e}
$$

Представим уравнение (7), в соответствии с рассуждениями авторов статьи [25], в виде:

$$
\ln \left(a_{e}-a_{t}\right)=\beta-k_{1} t
$$

Сравнение значений параметра $\beta$, найденных из графической зависимости $\ln \left(\mathrm{a}_{\mathrm{e}}-\mathrm{at}_{\mathrm{t}}\right)-\mathrm{t}$, с двумя возможными теоретическими интерпретациями ( $\left.\beta_{\mathrm{L} и} \beta_{\mathrm{D}}\right)$ позволяет сделать выводы о том, какая из стадий является скорость определяющей.

Значения $\beta_{\mathrm{D}}$ и $\beta_{\mathrm{L}}$ вычислены из экспериментальных значений ае по уравнениям (10) и (11). Полученные результаты приведены в таблице 4, из которой видно, что при температуре $293 \mathrm{~K}$ параметр $\beta$ ближе к параметру $\beta_{\mathrm{L}}$ (т.е. к значению lnae), определенному по уравнению (11), что свидетельствует о большей применимости модели, основанной на представлении сорбции как химической реакции, чем модели внутренней диффузии. При температуре $303 \mathrm{~K} \beta$ лежит между значениями $\beta_{\mathrm{L}}$ и $\beta_{\mathrm{D}}$, что может указывать на возможность использования обеих моделей для описания экспериментальных результатов. При температуре 313 К параметр $\beta$ ближе к параметру $\beta$ D , что может свидетельствовать о применимости для описания сорбции $\mathrm{Li}^{+}$при данной температуре модели внутренней диффузии. 
Таблица 4. Значения параметров $\beta, \beta_{\mathrm{L}}$ и $\beta_{\mathrm{D}}$, полученных из экспериментальных кинетических кривых сорбции $\mathrm{Li}^{+}$на $\mathrm{Al}(\mathrm{OH})_{3}$ при различных температурах

Table 4. Parameter values $\beta, \beta_{\mathrm{L}}$, and $\beta_{\mathrm{D}}$ obtained from experimental kinetic curves of Li sorption ${ }^{+}$ on $\mathrm{Al}(\mathrm{OH})_{3}$ at different temperatures

\begin{tabular}{|c|c|c|c|}
\hline $\mathrm{T}, \mathrm{K}$ & $\beta$ & $\beta_{\mathrm{L}}=\ln \mathrm{a}_{\mathrm{e}}$ & $\beta_{\mathrm{D}}=\ln \mathrm{a}_{\mathrm{e}}+\ln 6 / \pi^{2}$ \\
\hline 293 & 3.310 & 3.389 & 2.893 \\
303 & 3.084 & 3.351 & 2.854 \\
313 & 3.108 & 3.453 & 2.956 \\
\hline
\end{tabular}

В работе [26] отмечается, что описание кинетики сорбции в ограниченном объеме уравнением псевдо-второго порядка не говорит однозначно о том, что имеет место химическая кинетика, или диффузионная кинетика, осложненная взаимодействием типа сорбат-сорбент. В этой же работе отмечается, что для установления механизма сорбции необходимо использовать дополнительную информацию, в частности, по энергиям активации. Ранее [16] установлено, что энергия активации в интервале температур 293-303 и $303=-313 \mathrm{~K}$ при адсорбции $\mathrm{Li}^{+}$на $\mathrm{Al}(\mathrm{OH})_{3}$ из Берикейского рассола составляет 94 и 46 кДж/моль соответственно, что свидетельствует в пользу химической кинетики данного процесса.

\section{Заключение}

Обработка экспериментальных данных была проведена двумя методами: с

\section{Список литературы}

1. Коцупало Н.П., Рябцев А.Д., Болдырев В.В. // Наука в России. 2011. № 5. С. 28-31.

2. Кудрявцев П.Г. // Альтернативная энергетика и экология (ISJAEE). 2016. № 13-14. C. $72-88$.

3. Коцупало Н.П., Рябцев А.Д. Химия и технология получения соединений лития из литиеносного гидроминерального сырья. ЗАО «Экостар-Наутех». Новосибирск. Академ. изд-во «Гео». $2008.291 \mathrm{c.}$

4. Flexer V., Baspineiro C.F., Claudia Inés Galli C.I. // Science of the Total Environment. 2018. Vol. 639. pp. 1188-1204.

5. Саенко Е.В., Леонтьева Г.В., Вольхин В.В., Колышкин А.С. // Журнал неорганической химии. 2007. Т. 52. № 8. С.1399-1404.

6. Chitrakar R., Kanoh H., Makita Y., Miyai Y. et al. // J. Mater. Chem. 2000. Vol. 10. pp. 2325-2329. использованием уравнений внешней и внутренней диффузии и с применением расчетных кинетических диаграмм для смешанной диффузии. Последний выявил некоторое превалирование в смешанно-диффузионном механизме при 293 К внешней, а при 303 К и 313 К внутренней диффузии.

Описание кинетики сорбции $\mathrm{Li}^{+}$на $\mathrm{Al}(\mathrm{OH})_{3}$ с использованием моделей псевдо-первого и псевдо-второго порядков показывает, что при $293 \mathrm{~K}$ значение величины сорбции дает лучшее совпадение с экспериментальной, при использовании модели псевдо-первого порядка. При температурах $303 \mathrm{~K}$ и $313 \mathrm{~K}$ значение величин сорбции лучше совпадает с экспериментальными при использовании модели псевдо-второго порядка.

7. Chitrakar R., Kasaishi S., Umeno A., Sakane K. et al. // J. Solid State Chem. 2002. Vol. 169. pp. 35-43.

8. Ohashi F., Tai Y.// Materials Letters. 2019. Vol. 251. pp. 214-217.

9. Ventura S., Bhamidi S., Hornbostel M. // Proceedings, 43rd Workshop on Geothermal Reservoir Engineering Stanford University, Stanford, California, February 12-14. 2018. SGP-TR-213 pp. 1-5.

10.Song, J.F., Nghiem L.D., Li X.-M., He T. // Environmental Science: Water Research \& Technology. 2017. Vol. 3(4). pp. 593-597.

11.Рябцев А.Д. и др. Патент РФ. № 2455063 RU. 2012.

12.Комплексная переработка поликомпонентных литиеносных рассолов с предварительным их обогащением по литию / Под ред. Н.П. Коцупало; ЗАО «Экостар-Наутех». Новосибирск. Академ. изд-во «Гео». 2014. 172 с. 
13.Коцупало Н.П., Рябцев А.Д. Интеркаляционные соединения гидроксида алюминия с солями лития и их использование в промышленной практике. ООО «Экостар-Наутех». Новосибирск. Академ. изд-во «Гео».. 2016. $155 \mathrm{c}$.

14.Jiang H., Yang Y., Sun S., Yu J. // J. Chem. Eng. 2020. Vol. 98. pp. 544-555.

15.Рамазанов А.Ш. и др. Патент РФ. № 2660864 C2. 2018.

16.Рамазанов А.Ш. // Химия и технология воды. 1991. Т.13. № 2. С. 140-143.

17.Рамазанов А.Ш., Каспарова М.А., Сараева И.В., Алхасов А.Б. и др. // Экология и промышленность России. 2016. Т. 20. № 2. С.1417.

18.Гельферих Ф. Иониты. М. Изд-во иностр. лит. $1962.490 \mathrm{c.}$

19.Boyd G.E., Adamson A.W., Myers L.S. // J. Am. Chem. Soc. 1947. Vol. 69. pp. 2836-2848.
20.Веницианов Е.В. Рубинштейн Р.Н. Динамика сорбции из жидких сред. М. Наука. $1983.237 \mathrm{c}$.

21.Дресвянников А.Ф., Петрова Е.В., Цыганова М.А. // Журн. физ. химии. 2010. Т.84. С. 727-732.

22.Mathew A.P., Weber W.J. // Chem. Eng. Commun. 1984. Vol. 25. pp. 157-171.

23. Алосманов Р.М. // Вестн. Моск. Ун-та. Сер.2.Химия. 2011. Т. 52. № 2. С. 145-148.

24.Ho Y. // Water Res. 2006. Vol. 40. pp. 119125.

25.Rudzinski W., Plazinski W. // J. Phys. Chem. C. 2007. Vol. 111. pp. 15100-15110.

26.Хамизов Р.Х., Свешникова Д.А., Кучерова А.Е., Синяева Л.А. // Журн. физ. химии. 2018. T. 92. C.1451-1460.

\title{
The kinetics of sorption of lithium cationby freshly precipitated aluminium hydroxide from natural brine
}

\author{
(C) 2021 Ramazanov A.Sh. ${ }^{1,2}$, Sveshnikova D.A. ${ }^{1}$, Ataev D.R. ${ }^{1}$ \\ ${ }^{I}$ Institute of Geothermal and Renewable Energy Problems, Branch of the Joint Institute for High Tempera- \\ tures of the Russian Academy of Sciences, Makhachkala, Russian Federation \\ ${ }^{2}$ Dagestan State University, Makhachkala, Russian Federation
}

For over twenty years of the 21 st century, the consumption of lithium compounds in terms of lithium carbonate, used as a standard market commodity, has tripled, and the price has increased from $\$ 2$ to $\$ 8$ per kilogram. More than $35 \%$ of lithium compounds are used in the production of batteries and chemical power sources, about $32 \%$ in the production of ceramics and glass. With an increase in demand for lithium products, the raw material base has fundamentally changed; now, about $70 \%$ of lithium compounds are extracted from hydromineral raw materials. Lithium carbonate is obtained from strong sodium chloride type brines from the American continent using the halurgic technology. In China, from magnesium and calcium chloride type brines with a high lithium content and a total mineralization of more than $400 \mathrm{~g} / \mathrm{dm}^{3}$, lithium is extracted using a granular sorbent developed by ZAO Ecostar-Nautech (Novosibirsk, Russia).

This study is devoted to the investigation of the kinetics of sorption of $\mathrm{Li}^{+}$by freshly precipitated aluminium hydroxide from a natural lithium-containing sodium chloride calcium type brine with a mineralization of about $70 \mathrm{~g} / \mathrm{dm}^{3}$.

The kinetic curves of $\mathrm{Li}^{+}$adsorption on $\mathrm{Al}(\mathrm{OH})_{3}$ at different temperatures were processed by the equations of diffusion and chemical kinetics. The processing of experimental data using the calculated kinetic diagrams for mixed diffusion revealed the prevalence of external diffusion in the mixed diffusion mechanism at $293 \mathrm{~K}$, and at $303 \mathrm{~K}$ and $313 \mathrm{~K}$ internal diffusion prevailed.

The experimental data were also processed according to the pseudo-first and pseudo-second order equations used for the description of chemical kinetics. A comparison of the results showed that at $293 \mathrm{~K}$ the value of the calculated sorption value provides a better agreement with the experimental value when using the pseudo-first order model with practically the same correlation coefficients of $\mathrm{R}^{2}$ for both models. At temperatures of 303 and $313 \mathrm{~K}$, the sorption value was in better agreement with the experimental value when using the pseudo-second order model. The chemical kinetics of this process in the temperature ranges of 293-303 K and 303-313 K was evidenced by the activation energies of 94 and $46 \mathrm{~kJ} / \mathrm{mol}$, respectively.

Keywords: natural brine, lithium, aluminium hydroxide, sorption, kinetics. 


\section{References}

1. Kotsupalo N.P., Ryabtsev A.D., Boldyrev V.V., Nauka v Rossii, 2011, No 5, pp. 28-31.

2. Kudryavtsev P.G., Al'ternativnaya energetika $i$ ekologiya (ISJAEE), 2016, No 13-14, pp. 72-88.

3. Kotsupalo N.P., Ryabtsev A.D. Kkimiya i tekhnologiya polucheniya soedineniy litiya iz litienosnogo gidromineral'nogo sy-r'ya. ZAO «Ekostar-Nautekh». Novosi-birsk. Akadem. izdvo «Geo». 2008. 291 p.

4. Flexer V., Baspineiro C.F., Claudia Inés Galli C.I., Science of the Total Environment, 2018., Vol. 639, pp. 1188-1204.

5. Saenko E.V., Leont'eva G.V., Vol'khin V.V., Kolyshkin A.S., Zhurnal neorganicheskoy khimii, 2007, Vol. 52, No 8, pp. 13991404.

6. Chitrakar R., Kanoh H., Makita Y., Miyai Y. et al., J. Mater. Chem., 2000, Vol. 10, pp. 2325-2329.

7. Chitrakar R., Kasaishi S., Umeno A., Sakane K. et al., J. Solid State Chem., 2002, Vol. 169, pp. 35-43.

8. Ohashi F., Tai Y., Materials Letters, 2019, Vol. 251, pp. 214-217.

9. Ventura S., Bhamidi S., Hornbostel M., Proceedings, 43rd Workshop on Geothermal Reservoir Engineering Stanford University, Stanford, California, February 12-14, 2018, SGP-TR-213, pp. 1-5.

10.Song, J.F., Nghiem L.D., Li X.-M., He T., Environmental Science: Water Re-search \& Technology, 2017, Vol. 3(4), pp. 593-597.

11.Ryabtsev A.D. i dr. Patent RF. No 2455063 , RU. 2012.

12.Kompleksnaya pererabotka poli-komponentnykh litienosnykh rassolov $\mathrm{s}$ predvaritel'nym ikh obogashcheniem po litiyu,

Рамазанов Арсен Шамсудинович - профессор, д.Х.н., зав.лабораторией, институт проблем геотермии и возобновляемой энергетики филиала ОИВТ РАН, заведующий кафедрой аналитической и фармацевтической химии. Дагестанский государственный университет, Махачкала

Свешникова Джаннет Алексеевна - к.х.н., ведущий научный сотрудник, Институт проблем геотермии и возобновляемой энергетики филиала ОИВТ РАН, Махачкала

Атаев Давид Русланович - к.х.н., старший научный сотрудник, Институт проблем геотермии и возобновляемой энергетики филиала ОИВТ РАН, Махачкала
Pod red. N.P. Kotsupalo; ZAO «Eko-star-Nautekh». Novosibirsk. Akadem. izd-vo «Geo». 2014, 172 p.

13.Kotsupalo N.P., Ryabtsev A.D. In-terkalyatsionnye soedineniya gidroksida alyuminiya $\mathrm{s}$ solyami litiya i ikh ispol'zo-vanie v promyshlennoy praktike. OOO «Ekostar-Nautekh». Novosibirsk. Akadem. izd-vo «Geo».. 2016. 155 p.

14.Jiang H., Yang Y., Sun S., Yu J., J. Chem. Eng., 2020, Vol. 98, pp. 544-555.

15.Ramazanov A.SH. et al., Patent RF, No $2660864 \mathrm{~S} 2,2018$.

16. Ramazanov A.Sh., Khimiya i tekhnologiya vody, 1991, Vol. 13, No 2, pp. 140-143.

17.Ramazanov A.Sh., Kasparova M.A., Saraeva I.V., Alkhasov A.B. et al., Ekologiya $i$ promyshlennost' Rossii, 2016, Vol. 20, No 2, pp. 14-17.

18.Gel'ferikh F. Ionity, M., Izd-vo inostr. lit., 1962, $490 \mathrm{p}$.

19.Boyd G.E., Adamson A.W., Myers L.S., J. Am. Chem. Soc., 1947, Vol. 69, pp. 2836-2848.

20.Venitsianov E.V. Rubinshteyn R.N. Dinamika sorbtsii iz zhidkikh sred, M., Nauka, 1983, $237 \mathrm{p}$.

21.Dresvyannikov A.F., Petrova E.V., TSyganova M.A., Zhurn. fiz. khimii., 2010, Vol. 84, pp. 727-732.

22.Mathew A.P., Weber W.J., Chem. Eng. Commun., 1984, Vol. 25, pp. 157-171.

23.Alosmanov R.M., Vestn. Mosk. Un-ta. Ser.2.Khimiya, 2011, Vol. 52, No 2, pp. 145148.

24.Ho Y., Water Res., 2006, Vol. 40, pp. 119-125.

25.Rudzinski W., Plazinski W., J. Phys. Chem. C, 2007, Vol. 111, pp. 15100-15110.

26.KHamizov R.Kh., Sveshnikova D.A., Kucherova A.E., Sinyaeva L.A., Zhurn. fiz. Khimii, 2018, Vol. 92, pp.1451-1460

Ramazanov Arsen Sh. - Professor, Dr. Sci. (Chemistry), Head of the laboratory, Institute for Geothermy and Renewable Energy Problems. Branch of JIHT RAS. Head of department of Analytical and Pharmaceutical Chemistry, Dagestan State University, Makhachkala.a_ramazanov@mail.ru

Sveshnikova Dzhannet A. - Candidate of chemical sciences, Leading researcher. Institute for Geothermy and Renewable, Energy Problems. Branch of JIHT RAS, Makhachkala

Ataev David R. - Candidate of chemical sciences, Senior researcher. Institute for Geothermy and Renewable Energy Problems. Branch of JIHT RAS, Makhachkala 